\title{
Development of a Tandem Repeat-Based Polymerase Chain Displacement Reaction Method for Highly Sensitive Detection of 'Candidatus Liberibacter asiaticus'
}

\author{
Binghai Lou, Yaqin Song, Moytri RoyChowdhury, Chongling Deng, ${ }^{\dagger}$ Ying Niu, Qijun Fan, Yan Tang, \\ and Changyong Zhou ${ }^{\dagger}$
}

First author: College of Plant Protection, Southwest University, Chongqing 400715, P.R. China; first, second, fourth, fifth, sixth, and seventh authors: Guangxi Key Laboratory of Citrus Biology, Guangxi Academy of Specialty Crops, Guilin, Guangxi 541004, P.R. China; third author: Department of Biological Sciences, Idaho State University, 650 Memorial Dr., Pocatello 83201; and eighth author: Citrus Research Institute, Southwest University, Chongqing 400715, P.R. China.

Accepted for publication 10 October 2017.

\begin{abstract}
Huanglongbing (HLB) is one of the most destructive diseases in citrus production worldwide. Early detection of HLB pathogens can facilitate timely removal of infected citrus trees in the field. However, low titer and uneven distribution of HLB pathogens in host plants make reliable detection challenging. Therefore, the development of effective detection methods with high sensitivity is imperative. This study reports the development of a novel method, tandem repeat-based polymerase chain displacement reaction (TR-PCDR), for the detection of 'Candidatus Liberibacter asiaticus', a widely distributed HLB-associated bacterium. A uniquely designed primer set (TR2-PCDR-F/ TR2-PCDR-1R) and a thermostable Taq DNA polymerase mutant with strand

displacement activity were used for TR-PCDR amplification. Performed in a regular thermal cycler, TR-PCDR could produce more than two amplicons after each amplification cycle. Sensitivity of the developed TR-PCDR was 10 copies of target DNA fragment. The sensitive level was proven to be $100 \times$ higher than conventional PCR and similar to real-time PCR. Data from the detection of ' $\mathrm{Ca}$. L. asiaticus' with filed samples using the above three methods also showed similar results. No false-positive TR-PCDR amplification was observed from healthy citrus samples and water controls. These results thereby illustrated that the developed TR-PCDR method can be applied to the reliable, highly sensitive, and cost-effective detection of ' $\mathrm{Ca}$. L. asiaticus'.
\end{abstract}

Huanglongbing (HLB), also referred to as yellow shoot disease or greening disease, is one of the most serious diseases of citrus (Bové 2006). It was first reported in southern China in 1919 (Reinking 1919). Currently, HLB has been reported in more than 40 countries distributed in Asia, Africa, South America, North America, Central America, and Oceania (Coletta-Filho et al. 2004; Deng et al. 2008; Matos et al. 2013). Although the etiology of HLB remains unconfirmed because Koch's postulates have not been fulfilled, three gramnegative, phloem-limited fastidious liberibacters named 'Candidatus Liberibacter asiaticus', 'Ca. L. africanus' (Jagoueix et al. 1994; Murray and Stackebrandt 1995) and ' $\mathrm{Ca}$. L. americanus' (ColettaFilho et al. 2005; Teixeira et al. 2005b), respectively, were considered to be the causal agents for the disease (Wang and Trivedi 2013). Under natural conditions, ' $\mathrm{Ca}$. L. asiaticus' and ' $\mathrm{Ca}$. L. americanus' are mainly transmitted by Asian citrus psyllid Diaphorina citri (Halbert and Manjunath 2004; Teixeira et al. 2005a), while ' $\mathrm{Ca}$. L. africanus' is mainly transmitted by African citrus psyllid Trioza erytreae (Aubert 1987).

Integrated control management of HLB currently involves controlling psyllids in large areas, removing HLB-infected trees, and planting healthy nursery trees (Deng et al. 2016). Among these three steps, the effective removal of infected trees depends on an accurate

${ }^{\dagger}$ Corresponding authors: C. Deng; E-mail: cldeng88168@126.com; and C. Zhou; E-mail: zhoucy@swu.edu.cn

B. Lou and Y. Song made equal contributions to this study.

This work was supported by National Natural Science Foundation of China (31301635), Guangxi Natural Science Foundation (2015GXNSFCB139006), and Scientific Research and Technology Development Program of Guangxi (Gui Ke He 14123001-15 and Gui Ke AD16380046)

(c) 2018 The American Phytopathological Society diagnosis of HLB. During an early period, field characterization was the main diagnostic method for HLB. Leaf blotchy mottle was usually considered to be identification criteria for HLB infection in field diagnosis (McClean and Schwarz 1970). However, distinguishing leaf blotchy mottle symptoms from symptoms caused by nutrient deficiencies or other diseases was difficult (Li et al. 2009). Additionally, HLB has a potentially long incubation period before the infected citrus trees showed visual symptoms (Gottwald et al. 1989; Zhao 1981). The availability of molecular detection techniques directly targeting pathogens made the diagnosis of plant diseases efficient and easy. Several conventional polymerase chain reaction (PCR) methods targeting amplification of $16 \mathrm{~S}$ ribosomal DNA and ribosomal protein genes of the $\beta$-operon of HLB pathogens were developed for the detection of HLB pathogens (Hocquellet et al. 1999; Hung et al. 1999; Jagoueix et al. 1996; Teixeira et al. 2005a). However, low titer and uneven distribution of HLB pathogens in host plants made the incidence of detection using conventional PCR relatively low (Li et al. 2009; Louzada et al. 2016; Tatineni et al. 2008; Teixeira et al. 2008). Molecular approaches with higher sensitivity were required for reliable detection of HLB pathogens. Real-time PCR with sensitivity of $100 \times$ to $1,000 \times$ higher than that of conventional PCR was then developed for the detection of HLB pathogens (Li et al. 2006; Teixeira et al. 2008). However, real-time PCR requires expensive thermocyclers with a fluorescence detector which some laboratories cannot afford.

Most recently, loop-mediated isothermal amplification (LAMP) approaches for the detection of ' $\mathrm{Ca}$. L. asiaticus' were developed (Okuda et al. 2005; Rigano et al. 2014). LAMP utilized a Bacillus stearothermophilus DNA polymerase with chain displacement activity and could be performed in any regular thermal cycler or even in a water bath. The sensitivity level of LAMP for the detection of ' $\mathrm{Ca}$. L. asiaticus' was similar to that of real-time PCR (Rigano et al. 2014). However, products of LAMP were a mixture of 
stem-loop DNA with different lengths. Lack of a specific-size amplicon makes LAMP unreliable sometimes because it cannot distinguish between specific amplifications and nonspecific amplifications.

Therefore, the main objective of this study was to develop a method of overcoming the above mentioned drawbacks for the reliable and sensitive detection of ' $\mathrm{Ca}$. L. asiaticus'. The methodology developed and described in this article was named tandem repeat (TR)-based polymerase chain displacement reaction (PCDR). By using a TRbased primer set and a novel thermostable Taq DNA polymerase mutant with strand displacement activity, the developed TR-PCDR method could be performed for highly sensitive and specific detection of ' $\mathrm{Ca}$. L. asiaticus' in any regular thermal cycler. Sensitivity of the developed TR-PCDR was compared with conventional PCR and realtime PCR. Detection capability of the TR-PCDR was also evaluated with field samples. Furthermore, application prospects for TR-PCDR were discussed.

\section{MATERIALS AND METHODS}

Plant material and DNA extraction. Leaf samples were collected from 100 citrus trees with variable yellowing or mottling symptoms in an orchard in Guangxi, P.R. China. Each leaf sample consisted of eight leaves. From each tree, only one leaf sample was collected. An additional 36 leaf samples were collected from healthy citrus nursery trees at the Guangxi Academy of Specialty Crops (GASC), Guangxi, P.R. China. Leaves from mandarin (Citrus reticulata Blanco) trees infected by ' $\mathrm{Ca}$. L. asiaticus' and free of ' $\mathrm{Ca}$. L. asiaticus' kept in a screenhouse in GASC were used as sources of DNA for positive controls and negative controls, respectively.

Midribs were excised from each leaf sample and ground in liquid nitrogen. Total genomic DNA was extracted using a modified cetyltrimethylammonium bromide method from $100 \mathrm{mg}$ of the ground midrib tissue, as described previously (Lou et al. 2014). The DNA pellet was dissolved in $500 \mu$ of Tris-EDTA buffer $(10 \mathrm{mM}$ Tris- $\mathrm{HCl}$ and $1 \mathrm{mM} \mathrm{EDTA}, \mathrm{pH} 8.0$ ) and stored at $-20^{\circ} \mathrm{C}$ for later use.

Validation of TR locus. The complete genome sequence of 'Ca. L. asiaticus' strain Guangxi-1 (GenBank accession number CP004005) (Lin et al. 2013), excluding the prophage DNA sequence, was used to identify TR loci using Tandem Repeats Finder software (Benson 1999) with default parameters. The potential TR sites suitable for further design of forward primers were selected using the following criteria: period size (motif size) $\geq 25$, copy number $\geq 2.0$, percent matches $=100$, and percent indels $=0$. One candidate TR locus, ' $C a$. L. asiaticus' TR-002, was located by these analyses.

To ensure that the TR number (TRN) of ' $\mathrm{Ca}$. L. asiaticus' TR-002 was invariable in the ' $\mathrm{Ca}$. L. asiaticus' population, in silico sequence analysis was performed on each of all seven other genome sequences of ' $C a$. L. asiaticus' available in the GenBank database, including 'Ca. L. asiaticus' strain psy62 (CP001677), Ishi-1 (AP014595), A4 (CP010804), FL17 (JWHA00000000), HHCA (JMIL00000000), SGCA5 (LMTO00000000), and YCPsy (LIIM00000000). Additionally, the stability of the TRN of TR-002 was also evaluated by PCR analysis with primer set TR-V-F/TR-V-R (Table 1) flanking the tested TR locus. ' $\mathrm{Ca}$. L. asiaticus' isolates used for the PCR assay were those collected from China, as described previously (Islam et al. 2012). Procedures for PCR were similar to those described elsewhere (Lou et al. 2014), with an altered annealing temperature of $54^{\circ} \mathrm{C}$

TR-PCDR primer design. The structure of the primer set required by TR-PCDR is shown in Figure 1A. The sequence of the repeat motif of ' $\mathrm{Ca}$. L. asiaticus' TR-002 was directly used as forward primer. The reverse primer was designed using Primer-BLAST software (Ye et al. 2012) with parameters as follows: amplicon size $=$ 200 to $1,000 \mathrm{bp}$ and melting temperature $=61 \pm 1^{\circ} \mathrm{C}$. The specificity of the designed primer set to ' $\mathrm{Ca}$. L. asiaticus' was checked by in silico analysis through a BLASTn search against the GenBank nucleotide collection database. Finally, primer set TR2-PCDR-F/TR2-PCDR-1R
(Table 1) was obtained and used for the development of the TR-PCDR detection system of ' $\mathrm{Ca}$. L. asiaticus'.

TR-PCDR. TR-PCDR was carried out on an S1000 thermal cycler (Bio-Rad, Hercules, CA) using $20 \mu \mathrm{l}$ of reaction mixture containing $1 \times \mathrm{SD}$ polymerase reaction buffer, $3 \mathrm{mM} \mathrm{MgCl}_{2}, 375 \mu \mathrm{M}$ each dNTP, $0.5 \mu \mathrm{M}$ TR2-PCDR-F, $0.5 \mu \mathrm{M}$ TR2- PCDR-1R, $4 \mathrm{U}$ of SD Polymerase (BIORON, Ludwigshafen, Germany) (Ignatov et al. 2014), and $1 \mu$ of diluted DNA. The parameters for amplification were as follows: $92^{\circ} \mathrm{C}$ for $2 \mathrm{~min}$, followed by 35 cycles of $92^{\circ} \mathrm{C}$ for $30 \mathrm{~s}$ and $62^{\circ} \mathrm{C}$ for $2 \mathrm{~min}$, and a final incubation of $10 \mathrm{~min}$ at $62^{\circ} \mathrm{C}$. Each TR-PCDR run contained one negative control and one positive control. Amplification products were separated by electrophoresis in $1.5 \%$ agarose gels and visualized by staining with 4 S Red Plus Nucleic Acid Stain (Sangon Biotech, Shanghai, P.R. China).

Amplicon cloning and sequencing. The products of TRPCDR, a 234-bp DNA fragment with TRN of 1 and a 261-bp DNA fragment with TRN of 2, were cloned and sequenced as described previously (Lou et al. 2014). Additionally, plasmid DNA cloned with the 261-bp DNA fragment with TRN of 2 was isolated using the QIAprep Spin Miniprep Kit (Qiagen, Valencia, CA), and quantified with a Lambda 35 spectrophotometer (Perkin-Elmer, Waltham, MA). Copy number of target DNA per microliter of plasmid DNA suspension was calculated according to the available methodology (Lin et al. 2010).

Sensitivity of TR-PCDR and comparison with conventional PCR and real-time PCR. To evaluate the sensitivity of the newly developed methodology, TR-PCDR with primer set TR2PCDR-F/TR2-PCDR-1R was performed on a dilution series of plasmid DNA containing the 261-bp target sequence with a TRN of 2 . The plasmid DNA was diluted 10 -fold from $10^{5}$ to 1 copies $/ \mu 1$.

For comparison, the sensitivities of conventional PCR and realtime PCR were also evaluated. The conventional PCR was carried out with primer set OI1/OI2c (Table 1). Reaction conditions and parameters for conventional PCR were similar to those described elsewhere (Jagoueix et al. 1994). DNA templates for conventional PCR were serial dilutions of plasmid DNA cloned with a 1,168-bp target DNA fragment. The real-time PCR with primer set HLBas/ HLBr and probe HLBp (Table 1) was performed as described previously ( $\mathrm{Li}$ et al. 2006). DNA templates for real-time PCR were serial dilutions of plasmid DNA cloned with a 76-bp target DNA fragment. The plasmid DNA templates used for conventional PCR and real-time PCR were diluted as described above. All reactions for TR-PCDR, conventional PCR, and real-time PCR were performed in triplicate.

Detection of ' $\mathrm{Ca}$. L. asiaticus' with field samples. In total, 100 collected field samples mentioned above were used for the detection of ' $\mathrm{Ca}$. L. asiaticus' with the TR-PCDR method. Additionally, these samples were also analyzed using conventional PCR with primer sets OI1/OI2c (Table 1) and real-time PCR with primer set HLBas/HLBr and probe HLBp (Table 1). Cycle threshold values

TABLE 1. Information of primers and probe used in this study

\begin{tabular}{lll}
\hline $\begin{array}{l}\text { Primer or } \\
\text { probe }\end{array}$ & \multicolumn{1}{c}{ Sequence $\left(5^{\prime}-3^{\prime}\right)$} & \multicolumn{1}{c}{ Reference } \\
\hline $\begin{array}{l}\text { TR-V-F } \\
\text { TR-V-R }\end{array}$ & CCGATAGACCATCCTATGAGT & $\begin{array}{l}\text { This study } \\
\text { This study }\end{array}$ \\
$\begin{array}{l}\text { TR2- } \\
\text { PCDR-F }\end{array}$ & TGTCCATATGATCTTCGATAATGCGAG & This study \\
TR2- & AGGGTGGATTAGATCGTTTTCTCTCAA & This study \\
PCDR-1R & & Li et al. 2006 \\
HLBas & TCGAGCGCGTATGCAATACG & Li et al. 2006 \\
HLBr & GCGTTATCCCGTAGAAAAAGGTAG & Li et al. 2006 \\
HLBp & 6-FAM-AGACGGGTGAGTAACGCG- & \\
& BHQ-1 & Jagoueix et al. \\
OI1 & GCGCGTATGCAATACGAGCGGCA & 1994 \\
OI2c & GCCTCGCGACTTCGCAACCCAT & Jagoueix et al. \\
& & 1994 \\
\hline
\end{tabular}


derived from a real-time PCR assay were converted into numbers of bacteria per gram of midrib tissue using a grand universal regression equation $(Y=13.82-0.2866 X)(\mathrm{Li}$ et al. 2008, 2009) and three ribosomal RNA operons per cell of 'Ca. L. asiaticus' (Duan et al. 2009).

\section{RESULTS}

TR-PCDR primer design and validation. Successful design of the TR-PCDR primer set relied on identifying suitable TR loci (Fig. 1A). By sequence analyses, only one TR locus, ' $\mathrm{Ca}$. L. asiaticus' TR002 , spanning from nucleotide position 683,160 to 683,213 in the genome of ' $\mathrm{Ca}$. L. asiaticus' strain Guangxi-1 (CP004005), met the criteria required for the design of the forward primer of TR-PCDR. TR-002 possessed two perfect, tandem, 27-bp repeats (Fig. 1A). In silico pan-genomic analysis indicated that TRN of TR-002 in all ' $\mathrm{Ca}$. L. asiaticus' genomes available in the GenBank database were the same. Further PCR amplification of the TR-002 region with primer set TR-V-F/TR-V-R showed that the same size (143 bp) amplicon could be amplified from each analyzed ' $\mathrm{Ca}$. L. asiaticus' isolate collected from different areas of China (data not shown). These results robustly demonstrated that TRN of TR-002 was invariable among the ' $\mathrm{Ca}$. L. asiaticus' population, indicating that TR-002 was reliable for the design of the TR-PCDR forward primer.

Because the melting temperature of the 27-bp repeat unit of TR-002 was in the range of $61 \pm 1^{\circ} \mathrm{C}$, this 27-bp sequence was directly used as the forward primer TR2-PCDR-F. By using Primer-BLAST software, three paired reverse primers were designed. However, except for

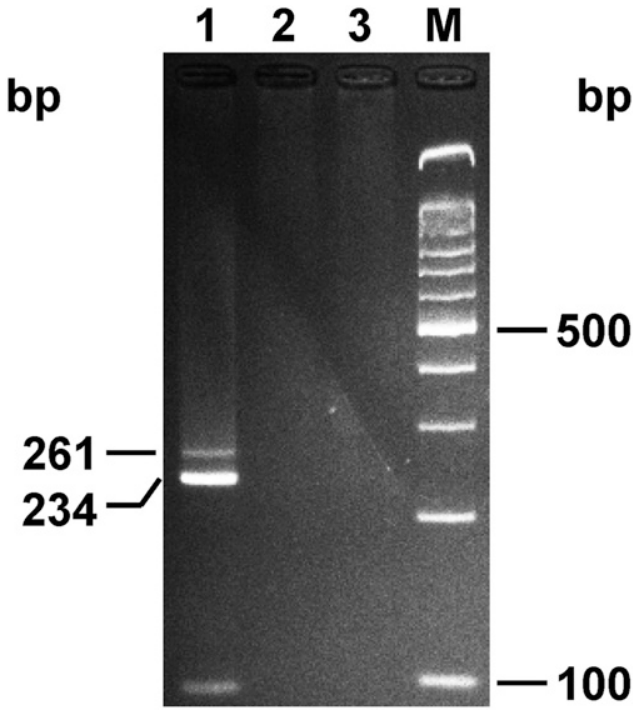

Fig. 2. Electrophoresis on $1.5 \%$ agarose gel of products amplified from different DNA templates by tandem repeat (TR)-based polymerase chain displacement reaction (PCDR) with primer set TR2-PCDR-F/TR2-PCDR-1R. Lane 1, positive DNA control extracted from 'Candidatus Liberibacter asiaticus'infected mandarin; lane 2, negative DNA control extracted from healthy mandarin; lane 3, water control; lane M, molecular standard ladder.

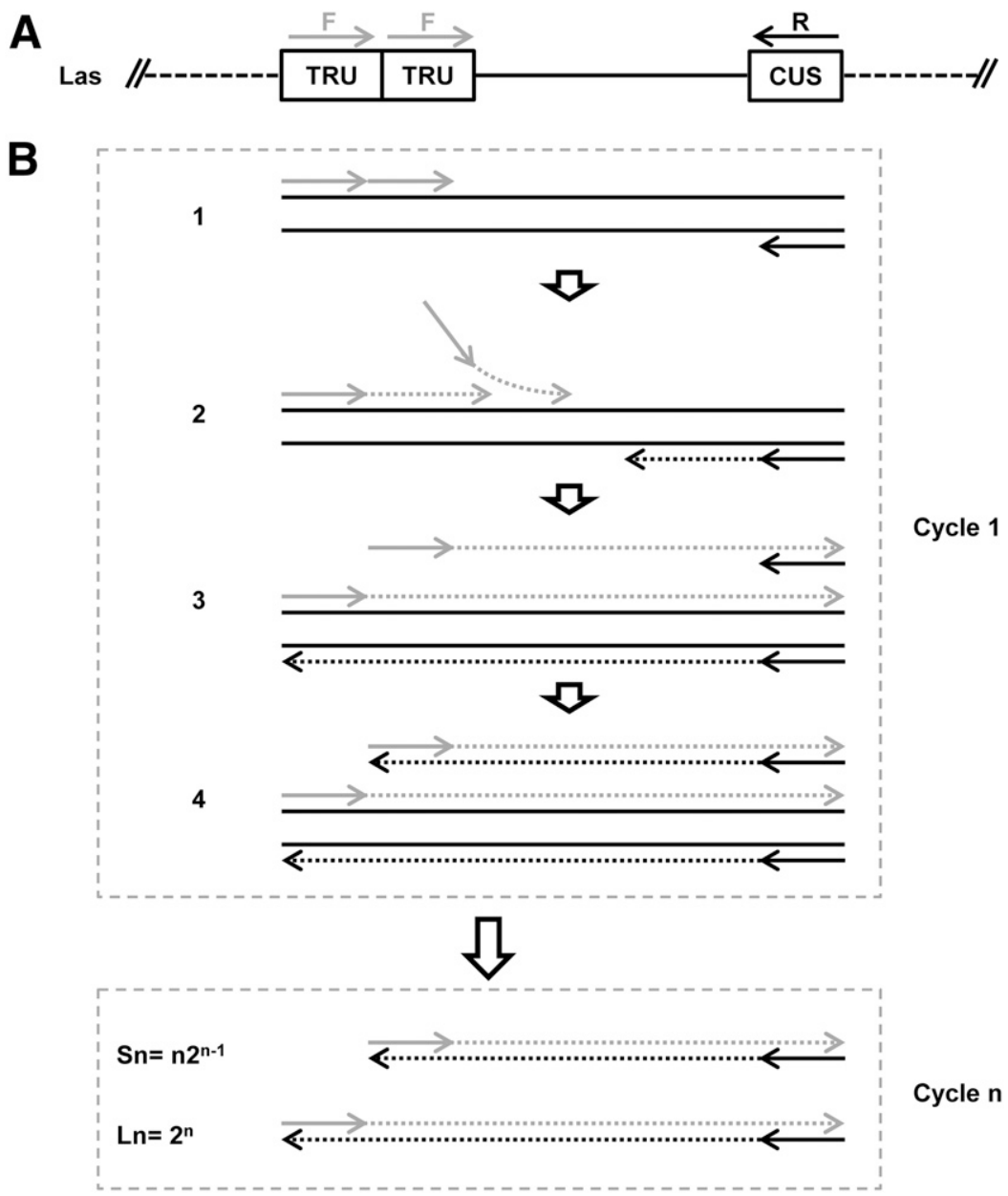

Fig. 1. Schematic representation of the mechanism of tandem repeat (TR)-based polymerase chain displacement reaction (PCDR). A, Schematic diagram of TRPCDR primer set design on the genome of 'Candidatus Liberibacter asiaticus'. B, Steps in the TR-PCDR reaction. Gray and black arrows represent forward and reverse primers, respectively. Rectangles labeled with TRU represent tandem repeats. The rectangle labeled with CUS represents conserved and unique sequence. Sn indicates copy number of the amplicon with a TR number (TRN) of 1 after $n$ cycles and Ln indicates copy number of the amplicon with a TRN of 2 after $n$ cycles. 
TR2-PCDR-1R, both of the other two designed reverse primers yielded nonspecific amplicons after TR-PCDR amplification when paired with TR2-PCDR-F (data not shown). When primer set TR2PCDR-F/TR2-PCDR-1R was used, two specific fragments, a 234-bp amplicon with TRN of 1 and a 261-bp amplicon with TRN of 2, could be reproducibly amplified from the positive control, whereas no amplicon was observed from negative control and water control (Fig. 2). Sequencing analysis indicated that the amplified two fragments were target amplicons. A further test with 36 DNA samples extracted from healthy citrus nursery trees also showed no amplification for TR-PCDR with primer set TR2-PCDR-F/TR2PCDR-1R. In silico specificity analysis confirmed that this primer set was only specific to ' $C a$. L. asiaticus'. These results indicated that TR-PCDR with primer set TR2-PCDR-F/TR2-PCDR-1R could be used for reliable detection of ' $\mathrm{Ca}$. L. asiaticus'.

Sensitivity of TR-PCDR. The sensitivity of TR-PCDR was evaluated and compared with conventional PCR and real-time PCR, respectively. Three 10 -fold dilution series from $10^{5}$ to 1 copies of plasmid DNA cloned with target DNA fragments were used as DNA templates for TR-PCDR, conventional PCR, and real-time PCR. For TR-PCDR, specific amplicons could be obtained from plasmid DNA templates containing $10^{5}$ to 10 copies of target DNA, indicating that the lowest detectable level of TR-PCDR was 10 copies of target DNA (Fig. 3A). For conventional PCR, positive reactions were only observed from plasmid DNA templates containing $10^{5}$ to $10^{3}$ copies of target DNA, suggesting that the sensitivity of conventional PCR was $10^{3}$ copies of target DNA (Fig. 3B). For real-time PCR, a fluorescence signal was accumulated from plasmid DNA templates containing $10^{5}$ to 10 copies of target DNA, indicating that the sensitivity of real-time PCR was 10 copies of target DNA (data not shown). These data demonstrated that the sensitive level of TR-PCDR was $100 \times$ higher than conventional PCR and similar to real-time PCR.

Evaluation of detection capability with field samples. In total, 100 leaf samples with variable yellowing or mottling symptoms were collected form a citrus orchard in Guangxi Province, P.R. China. Extracted genomic DNA from these field samples was used for the detection of ' $\mathrm{Ca}$. L. asiaticus' by TR-PCDR with primer set TR2-PCDR-F/TR2-PCDR-1R, conventional PCR with primer set OI1/OI2c, and real-time PCR with primer set HLBas/HLBr and probe HLBp. Among the 100 tested samples, 68 (68\%) were positive by TR-PCDR analysis, $52(52 \%)$ were positive by conventional PCR analysis, and $67(67 \%)$ were positive by realtime PCR analysis (Table 2). All of the 52 samples positive by conventional PCR were also positive by TR-PCDR and real-time PCR. Fifteen samples negative by conventional PCR were positive by
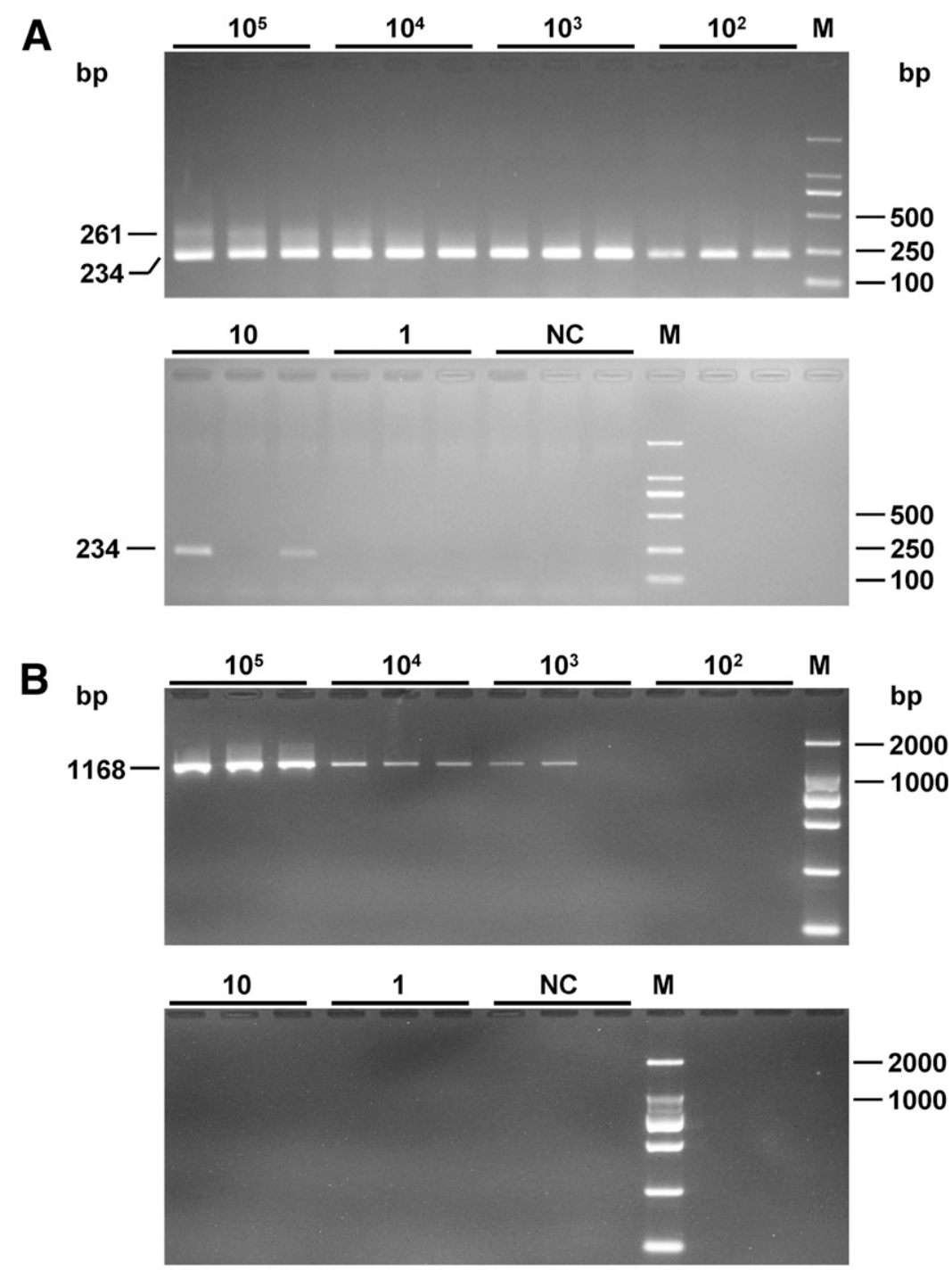

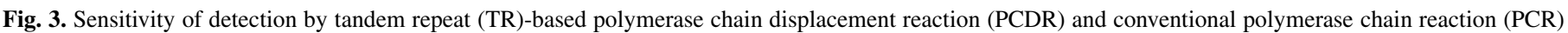

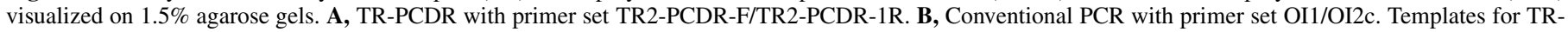

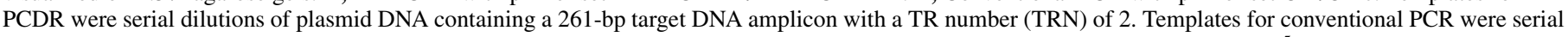

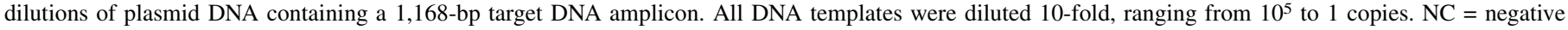
controls and $\mathrm{M}=$ molecular standard ladder. 
TR-PCDR (Fig. 4) and real-time PCR. Only one sample negative by conventional PCR was negative by real-time PCR but positive by TRPCDR (Table 2). This sample was further confirmed as a ' $C a$. L. asiaticus'-positive sample by sequencing amplicons obtained from TR-PCDR. These data confirmed that the sensitivity of TR-PCDR was indeed higher than conventional PCR and close to real-time PCR.

\section{DISCUSSION}

Early detection of HLB pathogens in citrus trees and timely removal of infected trees are considered to be key steps for effective control of HLB (Deng et al. 2016; Gottwald 2010; Wang et al. 2017). However, low titer and uneven distribution of HLB pathogens in infected trees make detection methods subject to false-negative results ( $\mathrm{Li}$ et al. 2009; Louzada et al. 2016; Tatineni et al. 2008; Teixeira et al. 2008). Therefore, the development of methodologies with high sensitivity for the detection of pathogens of HLB is of prime importance.

This study reported a TR-PCDR method for highly sensitive detection of ' $C a$. L. asiaticus', one of the presumed causal agents of HLB and widely distributed in the world (Bové 2006; Gottwald 2010). This methodology was developed from a previously reported method named PCDR (Harris et al. 2013), with modifications. By using multiple primers (three or four) and a polymerase with strand displacement activity but lacking 5' to $3^{\prime}$ exonuclease activity, PCDR could produce more than two amplicons after each amplification cycle. This made PCDR more sensitive than conventional PCR

TABLE 2. Detection of 'Candidatus Liberibacter asiaticus' from field samples by tandem repeat (TR)-based polymerase chain displacement reaction (PCDR) with primer set TR2-PCDR-F/TR2-PCDR-1R, conventional polymerase chain reaction $(\mathrm{C}-\mathrm{PCR})$ with primer set $\mathrm{OI} 1 / \mathrm{OI} 2 \mathrm{c}$, and real-time PCR with primer set $\mathrm{HLBas} / \mathrm{HLBr}$ and probe $\mathrm{HLBp}^{\mathrm{a}}$

\begin{tabular}{|c|c|c|c|c|c|}
\hline \multirow{2}{*}{$\begin{array}{l}\text { Detection } \\
\text { type }\end{array}$} & \multirow{2}{*}{$\begin{array}{c}\text { Samples } \\
(n)\end{array}$} & \multirow{2}{*}{$\begin{array}{c}\text { TR- } \\
\text { PCDR }\end{array}$} & \multirow{2}{*}{$\begin{array}{c}\text { C- } \\
\text { PCR }\end{array}$} & \multicolumn{2}{|r|}{ Real-time PCR } \\
\hline & & & & $\mathrm{Ct}$ value & Bacteria/g of midribs \\
\hline I & 52 & + & + & $\begin{array}{c}18.36- \\
30.14\end{array}$ & $2.53 \times 10^{8}-6.02 \times 10^{11}$ \\
\hline II & 15 & + & - & $\begin{array}{c}30.62- \\
34.17\end{array}$ & $1.77 \times 10^{7}-1.85 \times 10^{8}$ \\
\hline III & 1 & + & - & - & - \\
\hline IV & 32 & - & - & - & - \\
\hline Total & 100 & $\cdots$ & $\cdots$ & $\cdots$ & $\cdots$ \\
\hline
\end{tabular}

a Symbols: $\mathrm{Ct}=$ cycle threshold, $+=$ positive, and $-=$ negative.
(Harris et al. 2013). In TR-PCDR, we used only two primers to reduce interference effects between primers. However, to demonstrate PCDR using two primers, the former primer was designed on the TR region of the 'Ca. L. asiaticus' genome (Fig. 1A). Although a previous work had enhanced the sensitivity of real-time PCR detection of ' $C a$. L. asiaticus' by targeting TR on prophage DNA (Morgan et al. 2012), TR sites on prophage DNA in the ' $\mathrm{Ca}$. L. asiaticus' genome were not considered for the design of forward primers in this study. The existence of ' $\mathrm{Ca}$. L. asiaticus' strains without prophages (Katoh et al. 2014) or with only one (Zheng et al. 2016) of the two known prophages, SC1 and SC2 (Zhang et al. 2011), respectively, made the detection of ' $\mathrm{Ca}$. L. asiaticus' by targeting TR on prophage DNA possibly unreliable.

By employing primer pair TR2-PCDR-F and TR2-PCDR-1R, the developed TR-PCDR in this study could effectively detect ' $C a$. L. asiaticus' from plant extracts. The target amplification products of the TR-PCDR were two DNA fragments, a 234-bp amplicon with a TRN of 1 and a 261-bp amplicon with a TRN of 2 (Fig. 2). Theoretically, copy numbers of the 234-bp amplicon and 261-bp amplicon were $\mathrm{n} 2^{\mathrm{n}-1}$ and $2^{\mathrm{n}}$, respectively, after $n$ cycles of TR-PCDR amplification for each copy of original DNA fragment (Fig. 1B). The protocol of TR-PCDR in this study consisted of 35 cycles of amplification, which meant that the final number of the 234-bp amplicon was $17.5 \times$ of that of the 261-bp amplicon. This explained why, for some samples with a low titer of ' $\mathrm{Ca}$. L. asiaticus', only the 234-bp amplicon was observed on agarose gel. These data also indicated that the 234-bp amplicon should be treated as the main criterion of positive amplification for TR-PCDR with primer set TR2-PCDR-F/ TR2-PCDR-1R. No TR-PCDR amplicon was observed from any of the 36 analyzed DNA samples extracted from healthy citrus nursery trees, suggesting that the designed primer set TR2-PCDR-F/TR2PCDR-1R did not cross react with endophytes.

As a method which also could be performed in any regular thermal cycler, TR-PCDR was compared with conventional PCR in detection sensitivity. The results showed that TR-PCDR developed in this study was $100 \times$ more sensitive than conventional PCR (Fig. 3). Additionally, sensitivity of TR-PCDR was also compared with real-time PCR developed by Li et al. (2006). The results indicated that sensitivities of TR-PCDR and real-time PCR were similar. Further actual detection tests with a total of 100 filed samples also demonstrated that the sensitivity of TR-PCDR was indeed higher than conventional PCR and similar to real-time PCR (Table 2). These results suggested that the TR-PCDR method developed in this study

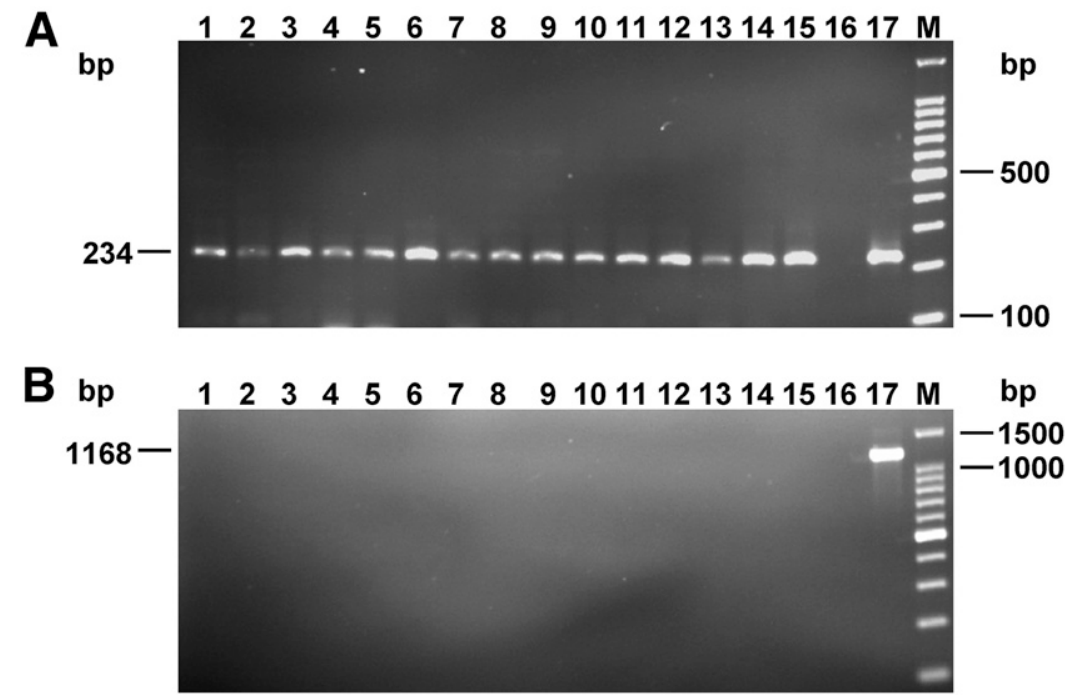

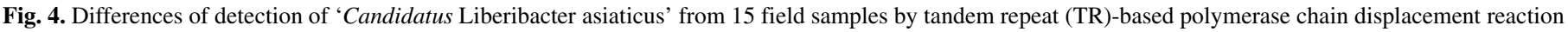

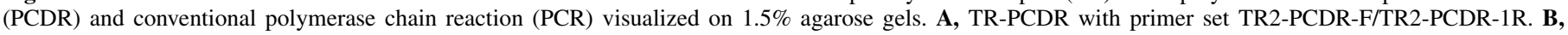

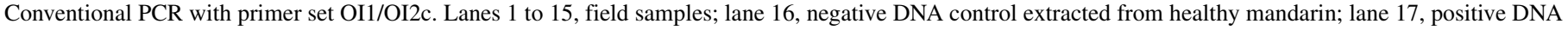
control extracted from ' $\mathrm{Ca}$. L. asiaticus'-infected mandarin; lane M, molecular standard ladder. 
could be used for highly sensitive detection of ' $\mathrm{Ca}$. L. asiaticus'. For each sample to be analyzed in the future, three replicates are suggested to be used in TR-PCDR detection because, like most of the other molecular detection methods, TR-PCDR also lacked amplification reproducibility at low target copy numbers (Fig. 3A).

The principle of the TR-PCDR method proposed in this study also can be used for development of detection techniques with high sensitivity for animal pathogens as well as other plant pathogens. After all, TR are commonly distributed in the genomes of bacteria, fungi, and viruses (Dutheil et al. 2016; Papageorgiou et al. 2016). Furthermore, inclusion of more than two repeats in the TR loci used for the development of new TR-PCDR methods can make the detection sensitivity higher.

In conclusion, a new methodology TR-PCDR was developed for the detection of ' $C a$. L. asiaticus' in this study. This method can be performed on any regular thermal cycler. The sensitivity of the developed TR-PCDR was 100× higher than conventional PCR and similar to real-time PCR. Field sample tests demonstrated the robustness of this methodology. More samples, especially those from other countries, need to be tested to further confirm the wide applicability of the TR-PCDR method for the detection of ' $\mathrm{Ca}$. L. asiaticus' in the future.

\section{LITERATURE CITED}

Aubert, B. 1987. Trioza erytreae del Guercio and Diaphorina citri Kuwayama (Homoptera: Psylloidea), the two vectors of citrus greening disease: $\mathrm{Bi}$ ological aspects and possible control strategies. Fruits 42:149-162.

Benson, G. 1999. Tandem repeats finder: A program to analyze DNA sequences. Nucleic Acids Res. 27:573-580.

Bové, J. M. 2006. Huanglongbing: A destructive, newly-emerging, century-old disease of citrus. J. Plant Pathol. 88:7-37.

Coletta-Filho, H. D., Takita, M. A., Targon, M. L. P. N., and Machado, M. A. 2005. Analysis of $16 \mathrm{~S}$ rDNA sequences from citrus huanglongbing bacteria reveal a different " $\mathrm{Ca}$. Liberibacter" strain associated with citrus disease in São Paulo. Plant Dis. 89:848-852.

Coletta-Filho, H. D., Targon, P. N., Takita, M. A., De Negri, J. D., Pompeu, J., Jr., Machado, M. A., do Amaral, A. M., and Muller, G. W. 2004. First report of the causal agent of huanglongbing ("Candidatus Liberibacter asiaticus") in Brazil. Plant Dis. 88:1382.

Deng, C., Bai, X., and Lou, B. 2016. Effects and experiences of integrated control of citrus huanglongbing performed in the last decade in Guangxi, P.R. China. South. Hortic. 27:26-27.

Deng, X., Chen, J., Feng, Z., Shan, Z., Guo, H., Zhu, J., Li, H., and Civerolo, E. L. 2008. Identification and characterization of the huanglongbing bacterium in pummelo from multiple locations in Guangdong, P.R. China. Plant Dis. 92: 513-518.

Duan, Y., Zhou, L., Hall, D. V., Li, W., Doddapaneni, H., Lin, H., Liu, L., Vahling, C. M., Gabriel, D. W., Williams, K. P., Dickerman, A., Sun, Y., and Gottwald, T. 2009. Complete genome sequence of citrus huanglongbing bacterium, 'Candidatus Liberibacter asiaticus' obtained through metagenomics. Mol. Plant-Microbe Interact. 22:1011-1020.

Dutheil, J. Y., Mannhaupt, G., Schweizer, G., Sieber, C. M. K., Münsterkötter, M., Güldener, U., Schirawski, J., and Kahmann, R. 2016. A tale of genome compartmentalization: The evolution of virulence cluster in smut fungi. Genome Biol. Evol. 8:681-704.

Gottwald, T. R. 2010. Current epidemiological understanding of citrus huanglongbing. Annu. Rev. Phytopathol. 48:119-139.

Gottwald, T. R., Aubert, B., and Zhao, X. 1989. Preliminary analysis of citrus greening (huanglongbing) epidemics in the People's Republic of China and French Reunion Island. Phytopathology 79:687-693.

Halbert, S. E., and Manjunath, K. L. 2004. Asian citrus psyllids (Sternorrhyncha: Psyllidae) and greening disease of citrus: A literature review and assessment of risk in Florida. Fla. Entomol. 87:330-353.

Harris, C. L., Sanchez-Vargas, I. J., Olson, K. E., Alphey, L., and Fu, G. 2013. Polymerase chain displacement reaction. Biotechniques 54:93-97.

Hocquellet, A., Toorawa, P., Bové, J. M., and Garnier, M. 1999. Detection and identification of the two Candidatus Liberobacter species associated with citrus huanglongbing by PCR amplification of ribosomal protein genes of the $\beta$ operon. Mol. Cell. Probes 13:373-379.

Hung, T. H., Wu, M. L., and Su, H. J. 1999. Development of a rapid method for the diagnosis of citrus greening disease using the polymerase chain reaction. J. Phytopathol. 147:599-604.

Ignatov, K. B., Barsova, E. V., Fradkov, A. F., Blagodatskikh, K. A., Kramarova, T. V., and Kramarov, V. M. 2014. A strong strand displacement activity of thermostable DNA polymerase markedly improves the results of DNA amplification. Biotechniques 57:81-87.

Islam, M. S., Glynn, J. M., Bai, Y., Duan, Y., Coletta-Filho, H. D., Kuruba, G., Civerolo, E. L., and Lin, H. 2012. Multilocus microsatellite analysis of 'Candidatus Liberibacter asiaticus' associated with citrus Huanglongbing worldwide. BMC Microbiol. 12:39.

Jagoueix, S., Bove, J. M., and Garnier, M. 1994. The phloem-limited bacterium of greening disease of citrus is a member of the $\alpha$ subdivision of the Proteobacteria. Int. J. Syst. Bacteriol. 44:379-386.

Jagoueix, S., Bové, J. M., and Garnier, M. 1996. PCR detection of the two 'Candidatus' Liberobacter species associated with greening disease of citrus. Mol. Cell. Probes 10:43-50.

Katoh, H., Miyata, S., Inoue, H., and Iwanami, T. 2014. Unique feature of a Japanese 'Candidatus Liberibacter asiaticus' strain revealed by whole genome sequencing. PLoS One 9:e106109.

Li, W., Hartung, J. S., and Levy, L. 2006. Quantitative real-time PCR for detection and identification of Candidatus Liberibacter species associated with citrus huanglongbing. J. Microbiol. Methods 66:104-115.

Li, W., Levy, L., and Hartung, J. S. 2009. Quantitative distribution of 'Candidatus Liberibacter asiaticus' in citrus plants with citrus huanglongbing. Phytopathology 99:139-144.

Li, W., Li, D., Twieg, E., Hartung, J. S., and Levy, L. 2008. Optimized quantification of unculturable Candidatus Liberibacter Spp. in host plant using real-time PCR. Plant Dis. 92:854-861.

Lin, H., Chen, C., Doddapaneni, H., Duan, Y., Civerolo, E. L., Bai, X., and Zhao, X. 2010. A new diagnostic system for ultra-sensitive and specific detection and quantification of Candidatus Liberibacter asiaticus, the bacterium associated with citrus huanglongbing. J. Microbiol. Methods 81:17-25.

Lin, H., Han, C. S., Liu, B., Lou, B., Bai, X., Deng, C., Civerolo, E. L., and Gupta, G. 2013. Complete genome sequence of a Chinese strain of "Candidatus Liberibacter asiaticus". Genome Announc. 1:e00184-12.

Lou, B., Bai, X., Bai, Y., and Deng, C., RoyChowdhury, M., Chen, C., and Song, Y. 2014. Detection and molecular characterization of a 16SrII-A* phytoplasma in grapefruit (Citrus paradise) with huanglongbing-like symptoms in China. J. Phytopathol. 162:387-395.

Louzada, E. S., Vazquez, O. E., Braswell, W. E., Yanev, G., Devanaboina, M., and Kunta, M. 2016. Distribution of 'Candidatus Liberibacter asiaticus' above and below ground in Texas citrus. Phytopathology 106:702-709.

Matos, L. A., Hilf, M. E., Chen, J., and Folimonova, S. Y. 2013. Validation of 'variable number of tandem repeat'-based approach for examination of 'Candidatus Liberibacter asiaticus' diversity and its applications for the analysis of the pathogen populations in the areas of recent introduction. PLoS One 8:e78994.

McClean, A. P. D., and Schwarz, R. E. 1970. Greening or blotchy-mottle disease of citrus. Phytophylactica 2:177-194.

Morgan, J. K., Zhou, L., Li, W., Shatters, R. G., Keremane, M., and Duan, Y.-P. 2012. Improved real-time PCR detection of 'Candidatus Liberibacter asiaticus' from citrus and psyllid hosts by targeting the intragenic tandemrepeats of its prophage genes. Mol. Cell. Probes 26:90-98.

Murray, R. G. E., and Stackebrandt, E. 1995. Taxonomic note: Implementation of the provisional status Candidatus for incompletely described procaryotes. Int. J. Syst. Bacteriol. 45:186-187.

Okuda, M., Matsumoto, M., and Tanaka, Y. 2005. Characterization of the tufB-secE-nusG-rplKAJL-rpoB gene cluster of the citrus greening organism and detection by loop-mediated isothermal amplification. Plant Dis. 89: $705-711$

Papageorgiou, K. V., Suárez, N. M., Wilkie, G. S., McDonald, M., Graham, E. M., and Davison, A. J. 2016. Genome sequence of canine herpesvirus. PLoS One 11:e0156015.

Reinking, O. A. 1919. Diseases of economic plants in southern China. Philipp. Agric. 8:109-134.

Rigano, L. A., Malamud, F., Ore, I. G., Filippone, M. P., Marano, M. R., do Amaral, A. M., Castagnaro, A. P., and Vojnov, A. A. 2014. Rapid and sensitive detection of Candidatus Liberibacter asiaticus by loop mediated isothermal amplification combined with a lateral flow dipstick. BMC Microbiol. 14:86

Tatineni, S., Sagaram, U. S., Gowda, S., Robertson, C. J., Dawson, W. O., Iwanami, T., and Wang, N. 2008. In planta distribution of 'Candidatus Liberibacter asiaticus' as revealed by polymerase chain reaction (PCR) and real-time PCR. Phytopathology 98:592-599.

Teixeira, D. C., Danet, J. L., Eveillard, S., Martins, E. C., de Jesus Junior, W. C., Yamamoto, P. T., Lopes, S. A., Bassanezi, R. B., Ayres, A. J., Saillard, C., and Bové, J. M. 2005a. Citrus huanglongbing in São Paulo State, Brazil: PCR detection of the 'Candidatus' Liberibacter species associated with the disease. Mol. Cell. Probes 19:173-179.

Teixeira, D. C., Saillard, C., Couture, C., Martins, E. C., Wulff, N. A., Eveillard-Jagoueix, S., Yamamoto, P. T., Ayres, A. J., and Bové, J. M. 2008. Distribution and quantification of Candidatus Liberibacter americanus, agent of huanglongbing disease of citrus in São Paulo State, Brasil, in leaves 
of an affected sweet orange tree as determined by PCR. Mol. Cell. Probes 22: $139-150$

Teixeira, D. C., Saillard, C., Eveillard, S., Danet, J. L., da Costa, P. I., Ayres, A. J., and Bové, J. 2005b. 'Candidatus Liberibacter americanus', associated with citrus huanglongbing (greening disease) in São Paulo State, Brazil. Int. J. Syst. Evol. Microbiol. 55:1857-1862.

Wang, N., Pierson, E. A., Setubal, J. C., Xu, J., Levy, J. G., Zhang, Y., Li, J., Rangel, L. T., and Martins, J. 2017. The Candidatus Liberibacter-host interface: Insight into pathogenesis mechanisms and disease control. Annu. Rev. Phytopathol. 55:451-482.

Wang, N., and Trivedi, P. 2013. Citrus huanglongbing: A newly relevant disease presents unprecedented challenges. Phytopathology 103:652-665.
Ye, J., Coulouris, G., Zaretskaya, I., Cutcutache, I., Rozen, S., and Madden, T. L. 2012. Primer-BLAST: A tool to design target-specific primers for polymerase chain reaction. BMC Bioinf. 13:134.

Zhang, S., Flores-Cruz, Z., Zhou, L., Kang, B. H., Fleites, L. A., Gooch, M. D., Wulff, N. A., Davis, M. J., Duan, Y. P., and Gabriel, D. W. 2011. 'Ca. Liberibacter asiaticus' carries an excision plasmid prophage and a chromosomally integrated prophage that becomes lytic in plant infections. Mol. Plant-Microbe Interact. 24:458-468.

Zhao, X. Y. 1981. Citrus yellow shoot (huanglongbing) in China-A review. Proc. Int. Soc. Citricult. 1:466-469.

Zheng, Z., Bao, M., Wu, F., Chen, J., and Deng, X. 2016. Predominance of single prophage carrying a CRISPR/cas system in "Candidatus Liberibacter asiaticus" strains in Southern China. PLoS One 11:e0146422. 